Mini Review

\title{
Metastatic disease or an early stage bladder cancer? a difficult question
}

\begin{abstract}
Bladder cancer is the second most common tumor in the urinary system. Compared to other malignancies, it has a relatively different metastatic potential. The skeletal system is the most common metastatic site of cancer. According to studies most common metastatic site of bladder cancer is skeletal system. In retrospective analysis; presence of bone metastasis was found to be more common in patients with deep muscle invasion than superficial muscular involvement and according to the histopathologic subgroup. Lymph node involvement, histologic grade, and $\mathrm{pT}$ stage were found to be prognostic factors for distant metastases. In contrast; there are cases with superficial tumors that develop metastasis in follow-up. Even if the patient was diagnosed at an early stage, we should be careful about bone metastasis that may develop in the follow-up.
\end{abstract}

Volume 8 Issue I - 2017

\section{Ozge Keskin, Esin Oktay}

Aydin Ataturk State Hospital,Turkey

\begin{abstract}
Correspondence: Ozge Keskin, MD,Aydin Ataturk State Hospital Medical Oncology Department, Aydin, Turkey, Tel 9.05E+ I I, Fax 9.03E+ I I, Email odurdu2000@yahoo.com
\end{abstract}

Received: February 28, 2017 | Published: April 28, 2017

Keywords: bladder cancer, bone, metastasis

Abbreviations: LN, lymph node; CT, computed tomography; TUR, transurethral resection; PET, positron emission tomography

\section{Introduction}

Bladder cancer is the second most common tumor in the urinary system. ${ }^{1}$ Compared to other malignancies, it has a relatively different metastatic potential and can metastasize to any organ. ${ }^{2}$ At the time of diagnosis; $70 \%$ of bladder cancer is not muscle-invasive. About $15-20 \%$ of these progress to muscle-invasive bladder cancer. ${ }^{3,4}$ The remaining $30 \%$ of patients have muscle-invasive local advanced or metastatic disease at the time of diagnosis. About $50 \%$ of those with muscle-invasive disease develop distant metastases even after radical cystectomy. ${ }^{5}$

The skeletal system is the most common metastatic site of cancer. ${ }^{6}$ Approximately $80 \%$ of advanced stage malignancies have

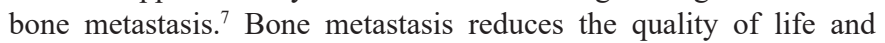
cause serious morbidity. Most common metastatic site of bladder cancer is skeletal system ${ }^{8,9}$ and this ratio is about $30 \% .^{10,11}$ In about $5-15 \%$ of these cases, only bone metastases were observed. ${ }^{12}$ The prognosis or pathogenesis of bone-predominant disease is not completely understood. According to Bianchi et al. ${ }^{13}$ analysis, elderly patients have only bone metastases. ${ }^{13}$ There are case reports of bone predominant disease with long survival while some case reports with short survival.

\section{Discussion}

In the case report of Ramos et al. ${ }^{14}$ patient had a muscle-invasive disease with no lymphovascular invasion. ${ }^{14}$ Radical cystectomy was performed after curative chemoradiotherapy. After the treatment, bone metastasis developed. The patient underwent local treatment for bone metastasis and was followed up for approximately 2 years. Similarly, the case of Joudi et al. ${ }^{15}$ had only-bone metastatic bladder cancer. The patient received systemic chemotherapy. Complete response was obtained with this treatment and radical curative surgery was performed. The patient was followed-up for 2 years with no evidence of disease. ${ }^{15}$

Taher et al. ${ }^{16}$ performed a retrospective analysis of 179 patients with bladder cancer. ${ }^{16}$ Approximately $65 \%$ of patients have clinical stage 3, 27\% have clinical stage 4 disease. According to pathological T stage; $76.2 \%$ of patients were pT3, $16.9 \%$ of patients were pT4 and $6.9 \%$ of patients were pT2 disease. The most frequent histologic grade was $2(58.7 \%)$. This was followed by grade $3(40.2 \%)$ and grade $1(1.1 \%)$ disease. Pelvic lymph node involvement was detected in $49.2 \%$ of the patients and nod negative disease was found in $50.8 \%$ of the patients. Deep muscle invasion was present in $61.5 \%$ of the patients whose disease was metastatic at the time diagnosis. Presence of bone metastasis was found to be statistically significant between patients with deep muscle invasion and superficial muscular involvement. There was also a difference in the development of bone metastasis according to the histopathologic subgroup. Of the patients with bone metastasis $92 \%$ had transitional cell carcinoma.

Squamous cell carcinoma was found in only about $8 \%$ of patients. In another study, more distant metastases were seen in transitional cell carcinoma of the bladder. But only transitional cell carcinoma histopathology was not found to be an independent prognostic factor. Lymph node (LN) involvement, histologic grade, and pT stage were also prognostic factors. ${ }^{8}{ }^{8}$ In Taher et al. ${ }^{16}$ 's analysis, isolated bone metastasis was most frequently observed. The most common regions were; pelvic bones and vertebral bodies. In this study, high histologic grade, high pT stage and LN involvement were also accepted as risk factors for bone metastasis. There was a significant difference in the development of bone metastasis between patients who had none of these risk factors and those who had more than one. ${ }^{16}$ In a retrospective analysis of Zaghloulis et al. ${ }^{8}$ pathologic stage, histological grade and pelvic LN involvement were found as independent factors affecting the development of distant metastases. ${ }^{8}$ Although disease progression depends on factors such as grade, tumor size and depth of invasion; according to some reports they are not sufficient to predict individual clinical outcomes. ${ }^{17,18}$

There are cases with superficial tumors in the literature that develop metastasis in follow-up ${ }^{19}$ Based on this, various theories have been considered; that patient has metastatic disease that we do not detect at the time of diagnosis, or the majority of cancer cells spread by lymphatic or vascular embolization. According to another hypothesis; there is micrometastasis at the time of diagnosis in superficial bladder cancer without muscle invasion, causing distant metastases in followup. ${ }^{20}$ In view of this, in order to predict the development of metastasis, 
bone turnover markers could be used in addition to radiological imaging in the follow-up (R). In patients with muscle-invasive disease, Taher et al. ${ }^{16}$ strongly recommended radiological examination of bone metastases. They also suggest that, the radical procedures may not be applied in patients with previously defined risk factors as these patients are likely to develop distant metastasis at follow-up. ${ }^{16}$

Unlike Taher et al.'s ${ }^{16}$ analysis, Shinagare et al. ${ }^{12}$ found that the most common site of metastasis is pelvic lymph nodes, while bone is the second most common site of metastasis for bladder cancer. ${ }^{12} \mathrm{~A}$ shorter metastasis free interval was seen at higher pT stage. However, there was no relationship between $\mathrm{pT}$ stage and any metastatic region. There was no difference between the metastatic regions in the histopathologic subtypes.

We also want to mention 2 cases of early stage bladder cancer without muscle invasive disease that we follow in our clinic.

\section{Case I}

A 72-year-old male was admitted to the urology clinic with hematuria on May, 2015. In abdominal ultrasonography and computed tomography (CT) scan of the abdomen, 2 nodular formation in the right lateral and posterior wall of the bladder were detected. No other pathology and paravesical lymph nodes were detected in CT scan. In CT scan of thorax, nodular density of $11 \times 7 \mathrm{~mm}$ in the right upper lobe of the lung was detected. Transurethral resection (TUR) of the bladder tumor was applied. Postoperative pathological report was pT1Nx transitional cell carcinoma with histological grade of 2 and without lymphovascular invasion. The patient developed pain in the neck. Cervical magnetic resonance imaging and positron emission tomography (PET)-CT showed a lytic bone lesion on $\mathrm{C} 4$ vertebra body. Vertebral operation was done. Carcinoma metastasis was seen in the postoperative pathology. Immunohistochemistry was negative for Synapsin A, cytokeratin 20, TTF-1, surfactant AA and positive for cytokeratin 7. Bladder cancer was considered as the primary tumor. Since the patient had metastatic disease, systemic chemotherapy treatment was decided after postoperative vertebral radiotherapy.

\section{Case 2}

A 71-year-old male patient was diagnosed with high grade transitional cell bladder carcinoma. pT1Nx disease was diagnosed with TUR-tumor. Radiologically there was no regional LN involvement or distant metastasis. Then patient underwent multiple intravesical BCG therapy. Six months after diagnosis, severe generalized bone pain developed and the patient was referred to our clinic. PET-CT was performed for staging. Multiple bone metastases and right pulmonary nodule was found. Patient underwent palliative radiotherapy for painful bone lesions and then, platinum-based chemotherapy and zoledronic acid was applied for metastatic disease.

\section{Conclusion}

As a result; even if the patient was diagnosed at an early stage, we should be careful and awake about bone metastasis that may develop in the follow-up. More attention must be paid to patients with the risk factors identified previously. In this respect, there is a need for larger studies for predictive markers of bone metastasis in bladder cancer.

\section{Funding}

None.

\section{Acknowledgements}

None.

\section{Conflicts of interests}

The authors declare that there is no conflict of interest.

\section{References}

1. Julian YP. Urinary tract cancers: An overview for general practice. Family Med Prim Care. 2016;5(3):533-538.

2. Shinagare AB, Ramaiya NH, Jagannathan JP, et al. Metastatic pattern of bladder cancer: correlation with the characteristics of the primary tumor. AJR Am J Roentgenol. 2011;196(1):117-122.

3. Clark PE, Agarwal N, Biagioli MC, et al. Bladder cancer. J Natl Compr Cancer Netw. 2013;11(4):446-475.

4. Peyromaure M, Zerbib M. T1G3 transitional cell carcinoma of the bladder: recurrence, progression and survival. BJU Int. 2004;93(1):6063.

5. Malkowicz SB, van Poppel H, Mickisch G, et al. Muscle-invasive urothelial carcinoma of the bladder. Urology. 2007;69(1 Suppl):3-16.

6. Poon M, Zeng L, Zhang L, Lam H, et al. Incidence of skeletal related events over time from solid tumour bone metastases reported in randomised trials using bone-modifying agents. Clin Oncol ( $R$ Coll Radiol). 2013;25(7):435-444.

7. Coleman R, Costa L, Saad F, et al. Consensus on the utility of bone markers in the malignant bone disease setting. Crit Rev Oncol Hematol. 2011;80(3):411-432.

8. Zaghloul MS. Distant metastasis from bilharzial bladder cancer. Cancer. 1996;77(4):743-749.

9. Sengelov L, Kamby C, Masse H. Pattern of metastases in relation to characteristics of primary tumor and treatment in patients with disseminated urothelial carcinoma. J Urol. 1996;155(1):111-114.

10. Saylor PJ, Armstrong AJ, Fizazi K, et al. New and emerging therapies for bone metastases in genitourinary cancers. Eur Urol. 2013;63(2):309320

11. Froehner M, Hölscher T, Hakenberg OW, et al. Treatment of bone metastases in urologic malignancies. Urol Int. 2014;93(3): 249-256.

12. Shinagare AB, Ramaiya NH, Jagannathan JP, et al. Metastatic pattern of bladder cancer: correlation with the characteristics of the primary tumor. AJR Am J Roentgenol. 2011;196(1):117-122.

13. Bianchi M, Roghmann F, Becker A, et al. Age-stratified distribution of metastatic sites in bladder cancer: A population-based analysis. Can Urol Assoc J. 2014;8(3-4):E148-E58.

14. Jorge D, Ramos, Heather H, Cheng, Evan Y, et al. Long-term survival in bone predominant metastatic urothelial carcinoma. Clin Genitourin Cancer. 2014;12(6):e241-e244.

15. Joudi FN, Dahmoush L, Spector DM, et al. Complete response of bony metastatic bladder urothelial cancer to neoadjuvant chemotherapy and cystectomy. Urol Oncol. 2006;24(5):403-406.

16. Taher AN, Kotb HM. Bone Metastases in Muscle Invasive Bladder Cancer. Journal of the Egyptian Nat. 2006;18(3):203-208.

17. Brausi M, Witjes JA, Lamm D, et al. A review of current guidelines and best practice recommendations for the management of nonmuscle invasive bladder cancer by the International Bladder Cancer Group. $J$ Urol. 2011;186(6):2158-2167. 
18. Jeong Hee Hong. Early isolated bone metastases without local recurrence in non-muscle invasive bladder cancer. Int J Surg Case Rep. $2015 ; 10: 41-44$

19. Matthews PN, Madden M, Bidgood KA, et al. The clinicopathological features of metastatic superficial papillary bladder cancer. $J$ Urol. 1984;132(5):904-906.
20. Alcaraz A, González-López R, Morote J, et al. Biochemical markers of bone turnover and clinical outcome in patients with renal cell and bladder carcinoma with bone metastases following treatment with zoledronic acid: The TUGAMO study. Br J Cancer. 2013;109(1): 121-130. 